\title{
EVERY SEMIPRIMARY RING IS THE ENDOMORPHISM RING OF A PROJECTIVE MODULE OVER A QUASI-HEREDITARY RING
}

\author{
VLASTIMIL DLAB AND CLAUS MICHAEL RINGEL
}

(Communicated by Donald S. Passman)

\begin{abstract}
The paper provides a proof of the following statement: Given a semiprimary ring $R$, there is a quasi-hereditary ring $A$ and an idempotent $e \in A$ such that $R \simeq e A e$.
\end{abstract}

In his Queen Mary College lectures [A], M. Auslander has shown that any left artinian ring $R$ occurs as the endomorphism ring of a projective $A$-module $P_{A}$ where $A$ is a semiprimary ring of finite global dimension. This ring $A$ is constructed as follows: let $N$ be the radical of $R$, let $n$ be its nilpotency index, and $M_{R}=\bigoplus_{i=1}^{n}\left(R / N^{i}\right)_{R}$; take $A=\operatorname{End}\left(M_{R}\right)$. Clearly, there is an idempotent $e$ of $A$ with $R=e A e$; thus $R$ is the endomorphism ring of the projective $A$-module $e A$. Hence the main step of the proof is the verification that $A$ has global dimension at most $n$. In case that $R$ is an Artin algebra, also $A$ is an Artin algebra. As Auslander has stressed, his result asserts that the Artin algebras of finite global dimension determine all Artin algebras.

The aim of this note is to show, that the so constructed ring $A$ is, in fact, quasi-hereditary in the sense of Cline-Parshall-Scott [S], [CPS]. For the definition and properties of quasi-hereditary rings we refer to [CPS], [PS], and also to $\left[D R_{1}\right]$; we point out, in particular, that they always have finite global dimension. We recall that the notion of a quasi-hereditary ring was introduced in order to have available a class of rings whose bounded derived categories can be built up from the bounded derived categories of division rings using "recollement".

Actually, the ring $R$ we will start with may be an arbitrary semiprimary ring. Let $N$ be its radical, $n$ the nilpotency index of $N$, and $M_{R}=\bigoplus_{i=1}^{n}\left(R / N^{i}\right)_{R}$. We consider $A=\operatorname{End}\left(M_{R}\right)$; this is again a semiprimary ring. Let $J_{t}$ be the set of all endomorphisms $\varphi$ of $M_{R}$ which factor through a module in $\operatorname{add}\left(\bigoplus_{i=1}^{t} R / N^{i}\right)_{R} ;$ this is obviously an ideal of $A$.

Theorem. The chain of ideals $0=J_{o} \subseteq J_{1} \subseteq \cdots \subseteq J_{n}=A$ is a hereditary chain, thus $A$ is quasi-hereditary.

Received by the editors May 11, 1988.

1980 Mathematics Subject Classification (1985 Revision). Primary 16A46, 16A65; Secondary $16 \mathrm{~A} 45$. 
As a consequence, we see that any semiprimary ring is the endomorphism ring of a projective module over a quasi-hereditary ring. In this sense, the quasi-hereditary rings determine all semiprimary rings.

The proof of the theorem is similar to our construction of a heredity chain in an Auslander algebra [DR 2 ]. The common criterion for the construction of heredity ideals in factor rings of endomorphism rings is formulated in $\S 1$, and $\S 2$ gives the proof of the theorem.

\section{HEREDITY IDEALS IN FACTOR RINGS OF ENDOMORPHISM RINGS}

In this section we consider modules over an arbitrary ring $R$. Given a module $M$, the direct sum of $c$ copies of $M$ will be denoted by $c M$, and add $M$ denotes the category of all direct summands of finite direct sums of $M$. A homomorphism $\alpha: X \rightarrow Y$ is said to factor through add $M$ provided it factors through a module in add $M$.

Proposition. Let $M^{\prime}, M, M^{\prime \prime}$ be modules with semiprimary endomorphism rings. Assume the following two conditions are satisfied:

(a) If $X, Y$ are indecomposable direct summands of $M$, and $\gamma: Y \rightarrow X$ is a noninvertible map, then $\gamma$ factors through add $M^{\prime}$.

(b) If $X, Y$ are indecomposable direct summands of $M \oplus M^{\prime \prime}$, and $\delta: Y \rightarrow C$, $\gamma: C \rightarrow X$ are maps with $C \in$ add $M$ such, that $\gamma \delta$ factors through add $M^{\prime}$, then there exists a decomposition

$$
C=C_{1} \oplus C_{2}, \gamma=\left[\gamma_{1}, \gamma_{2}\right], \delta=\left[\begin{array}{l}
\delta_{1} \\
\delta_{2}
\end{array}\right]
$$

such that both $\gamma_{1}$ and $\delta_{2}$ factor through add $M^{\prime}$.

Let $A=\operatorname{End}\left(M^{\prime} \oplus M \oplus M^{\prime \prime}\right)$, let $J^{\prime}$ be the set of elements of $A$ which factor through add $M^{\prime}$, and $J$ be the set of elements of $A$ which factor through $\operatorname{add}\left(M^{\prime} \oplus M\right)$. Then $J / J^{\prime}$ is a heredity ideal of $A / J^{\prime}$.

Proof. Let $\bigoplus_{i=1}^{p} M_{i}$. be a direct summand of $M$ such that $M_{1}, \ldots, M_{p}$ are pairwise nonisomorphic indecomposable modules and not isomorphic to direct summands of $M^{\prime}$ and such that $\operatorname{add}\left(M^{\prime} \oplus \bigoplus_{i=1}^{p} M_{i}\right)=\operatorname{add}\left(M^{\prime} \oplus M\right)$. We may assume that $M=\bigoplus_{i=1}^{p} M_{i}$ by replacing $M^{\prime \prime}$ by $M^{\prime \prime} \oplus \widetilde{M}$, where $\widetilde{M}$ is a direct complement of $\bigoplus_{i=1}^{p} M_{i}$ in $M$. Let $e_{i}$ be the projection of $M^{\prime} \oplus$ $M \oplus M^{\prime \prime}=M^{\prime} \oplus\left(\bigoplus_{i=1}^{p} M_{i}\right) \oplus M^{\prime \prime}$ onto $M_{i}$, and $e=\sum_{i=1}^{p} e_{i}$; furthermore, $e^{\prime}$ be the projection of $M^{\prime} \oplus M \oplus M^{\prime \prime}$ onto $M^{\prime}$. Note that $e^{\prime}, e_{1}, \ldots, e_{p}$ are orthogonal idempotents, and that $e_{1}, \ldots, e_{p}$ are pairwise inequivalent and primitive. We have $J^{\prime}=A e^{\prime} A, J=A\left(e^{\prime}+e\right) A$. Consider the residue ring $\bar{A}=A / J^{\prime}$, and denote the residue class of $\alpha \in A$ in $\bar{A}$ by $\bar{\alpha}$; a similar notation will be used for subsets of $A$. Clearly, $\bar{J}$ is idempotent, and $\bar{J}=\bar{A} \bar{e} \bar{A}$. It follows from condition (a) that $\bar{e} \bar{A} \bar{e}$ is the product of the division rings $\bar{e}_{i} \bar{A} \bar{e}_{i} \simeq$ $\operatorname{End}\left(M_{i}\right) / \operatorname{rad} \operatorname{End}\left(M_{i}\right)$ for $1 \leq i \leq p ;$ thus $\bar{e} \bar{A} \bar{e} \simeq \operatorname{End}(M) / \operatorname{rad} \operatorname{End}(M)$ and $\bar{e} \operatorname{rad} \bar{A} \bar{e}=0$. It remains to be seen that the multiplication map $\bar{A} \bar{e} \bigotimes_{\bar{e} \bar{A} \bar{e}} \bar{e} \bar{A} \rightarrow$ $\bar{A} \bar{e} \bar{A}$ is injective. 
Let $X, Y$ be indecomposable direct summands of $M \oplus M^{\prime \prime}$ and $e_{X}, e_{Y}$ the corresponding idempotents of $A$. It is sufficient to show that the multiplication map

$$
\bar{e}_{X} \bar{A} \bar{e} \bigotimes_{\bar{e} \bar{A} \bar{e}} \bar{e} \bar{A} \bar{e}_{Y} \rightarrow \bar{e}_{X} \bar{A} \bar{e} \bar{A} \bar{e}_{Y}
$$

is injective. The elements in $\bar{e}_{X} \bar{A} \bar{e} \bigotimes_{\bar{e} \bar{A} \bar{e}} \bar{e} \bar{A}_{Y}$ are of the form $u=\sum_{j=1}^{c} \bar{\gamma}_{j} \otimes \bar{\delta}_{j}$ where $\gamma_{j} \in e_{X} A e=\operatorname{Hom}(M, X)$ and $\delta_{j} \in e A e_{Y} \in \operatorname{Hom}(Y, M)$. Let $C=c M$,

$$
\gamma=\left[\gamma_{1}, \ldots, \gamma_{c}\right]: C \rightarrow X \quad \text { and } \quad \delta=\left[\begin{array}{c}
\delta_{1} \\
\vdots \\
\delta_{c}
\end{array}\right]: Y \rightarrow C .
$$

The multiplication map sends $u=\sum \bar{\gamma}_{j} \otimes \bar{\delta}_{j}$ to $\sum \bar{\gamma}_{j} \bar{\delta}_{j}=\overline{\gamma \delta}$. Assume now that $\overline{\gamma \delta}=0$, thus $\gamma \delta$ factors through add $M^{\prime}$. Therefore the condition (b) asserts that there is a decomposition $C=C_{1} \oplus C_{2}, \gamma=\left[\gamma_{1}, \gamma_{2}\right], \delta=\left[\begin{array}{l}\delta_{1} \\ \delta_{2}\end{array}\right]$ such that both $\gamma_{1}$ and $\delta_{2}$ factor through add $M^{\prime}$. Let $\omega_{1}$ be the projection of $C$ onto $C_{1}$, thus $\omega_{2}=1-\omega_{1}$ is the projection of $C$ onto $C_{2}$. Let $l_{j}: M \rightarrow c M$ be the $j$ th inclusion, and $\varepsilon_{j}: c M \rightarrow M$ the $j$ th projection. Thus $\gamma_{j}=\gamma l_{j}$, and $\delta_{j}=\varepsilon_{j} \delta$. By the lemma in [DR 2 ]

$$
\sum_{j=1}^{c} \gamma_{j} \otimes \delta_{j}=\sum_{j=1}^{c} \gamma \omega_{1} l_{j} \otimes \varepsilon_{j} \omega_{1} \delta+\sum_{j=1}^{c} \gamma \omega_{2} l_{j} \otimes \varepsilon_{j} \omega_{2} \delta
$$

in $\operatorname{Hom}(M, X) \otimes_{\operatorname{End}(M)} \operatorname{Hom}(Y, M)=e_{X} A e \otimes_{e A e} e A e_{Y}$. Since $\gamma \omega_{1}$ factors through add $M^{\prime}$, it follows that $\gamma \omega_{1} l_{j}$ belongs to $e_{X} J^{\prime} e$, thus the first summand on the right becomes zero in $\bar{e}_{X} \bar{A} \bar{e} \bigotimes_{\bar{e} \bar{A} \bar{e}} \bar{e} \bar{A} \bar{e}_{Y}$. Similarly, $\omega_{2} \delta$ factors through add $M^{\prime}$, and therefore also the second summand becomes zero in $\bar{e}_{X} \bar{A} \bar{e} \otimes_{\bar{e}} \bar{A} \bar{e}$ $\bar{e} \bar{A} \bar{e}_{Y}$. Consequently $u=0$. This completes the proof.

\section{Proof of THE THEOREM}

Let $R$ be semiprimary ring, $N$ its radical, and $N^{n}=0$. Note that for any $i$, the endomorphism ring $\operatorname{End}\left(R / N^{i}\right)_{R}$ is just $R / N^{i}$, thus semiprimary. It follows that the endomorphism ring $A$ of $\bigoplus_{i=1}^{n}\left(R / N^{i}\right)_{R}$ is semiprimary. Let $J_{t}$ be the ideal of all elements of $A$ which factor through $\bigoplus_{i=1}^{t}\left(R / N^{i}\right)_{R}$. We claim that $J_{t} / J_{t-1}$ is a hereditary ideal of $A$, for any $1 \leq t \leq n$. We want to apply the criterion of $\S 1$. Let

$$
M^{\prime}=\bigoplus_{i=1}^{t-1}\left(R / N^{i}\right)_{R}, M=\left(R / N^{t}\right)_{R}, M^{\prime \prime}=\bigoplus_{i=t+1}^{n}\left(R / N^{i}\right)_{R} .
$$

The indecomposable direct summands of $M$ are of the form $e R / e N^{t}$ with a primitive idempotent $e$ of $R$, those of $M^{\prime \prime}$ are of the form $e R / e N^{i}$ with a primitive idempotent $e$ of $R$, and $i>t$. The modules in add $M$ are just the 
projective $R / N^{t}$-modules. Also, note that $M^{\prime}$ is annihilated by $N^{t-1}$. We are going to verify the two conditions (a) and (b).

(a) Let $e_{1}, e_{2}$ be primitive idempotents of $R$, and $\gamma: e_{1} R / e_{1} N^{t} \rightarrow e_{2} R / e_{2} N^{t}$ a noninvertible map. Note that $\gamma$ cannot be surjective, since both $e_{i} R / e_{i} N^{t}$, $i=1,2$, are indecomposable projective $R / N^{t}$-modules. Thus the image of $\gamma$ is contained in $e_{2} N / e_{2} N^{t}$ and therefore annihilated by $N^{t-1}$. It follows that $\gamma$ can be factored through $e_{1} R / e_{1} N^{t-1}$ which belongs to add $M^{\prime}$.

(b) Again, let $e_{1}, e_{2}$ be primitive idempotents of $R$, and $X=e_{1} R / e_{1} N^{i}$, $Y=e_{2} R / e_{2} N^{j}$ for some $i, j \geq t$. Let $C$ be a projective $R / N^{t}$-module. Let $\delta: Y \rightarrow C, \gamma: C \rightarrow X$ be maps such that $\gamma \delta$ factors through add $M^{\prime}$. First, assume that the image $\delta\left(e_{2}\right)$ of $e_{2}$ under $\delta$ is annihilated by $N^{t-1}$. Then $e_{2} N^{t-1}$ is in the kernel of $\delta$, thus $\delta$ factors through the module $e_{2} R / e_{2} N^{t-1} \in$ add $M^{\prime}$. In this case, take $C_{1}=0$, and $C_{2}=C$.

It remains to consider the case that $\delta\left(e_{2}\right) N^{t-1} \neq 0$. In this case, $C_{1}=\delta\left(e_{2}\right) R$ is a projective $R / N^{t}$-module which is a direct summand of $C$. For, let $C=$ $\bigoplus U_{i}$ with indecomposable modules $U_{i}$, and with projections $\pi_{i}: C \rightarrow U_{i}$. Then, there exists $i$ with $\pi_{i} \delta\left(e_{2}\right) N^{t-1} \neq 0$. Now, $U_{i}$ is an indecomposable projective $R / N^{t}$-module, thus $U_{i} N$ is its unique maximal submodule. Since $\pi_{i} \delta\left(e_{2}\right)$ is not contained in $U_{i} N$, it follows that $\pi_{i} \delta: Y \rightarrow U_{i}$ is surjective. The image $C_{1}=\delta\left(e_{2}\right) R$ of $\delta$ is a local $R$-module which is annihilated by $N^{t}$ and which is mapped under $\pi_{i}$ onto the indecomposable projective $R / N^{t}$-module $U_{i}$. This shows that $C_{1}$ itself is projective and that the inclusion map $C_{1} \rightarrow C$ is a split monomorphism.

Let $C_{2}$ be a direct complement of $C_{1}$ in $C$; write $\gamma=\left[\gamma_{1}, \gamma_{2}\right], \delta=\left[\begin{array}{c}\delta_{1} \\ \delta_{2}\end{array}\right]$. Then $\delta_{2}=0$, thus it factors through add $M^{\prime}$. Since $\gamma \delta$ factors through add $M^{\prime}$, the image of $\gamma \delta$ is annihilated by $N^{t-1}$. But $\gamma \delta\left(e_{2}\right) \cdot N^{t-1}=0$ implies that $\delta\left(e_{2}\right) N^{t-1}$ is contained in the kernel of $\gamma$. Now, $\delta\left(e_{2}\right) R$ is isomorphic to $e_{2} R / e_{2} N^{t}$, thus $\gamma_{1}$ factors through $e_{2} R / e_{2} N^{t-1} \in \operatorname{add} M^{\prime}$.

This completes the proof of the theorem.

Remark. The ring $A=\operatorname{End}\left(M_{R}\right)$ is usually not basic, even if the ring $R$ is basic. The reason is the following one: In case that $R$ is basic, each non-projective indecomposable summand occurs in a direct decomposition of $M_{R}$ with multiplicity 1 , whereas each indecomposable projective $R$-module of Loewy length $l$ occurs with multiplicity $n-l+1$. Deleting the repeated copies of the indecomposable projective summands of $M_{R}$ one obtains a module whose endomorphism ring is basic and Morita equivalent to $A$.

\section{REFERENCES}

[A] M. Auslander, Representation dimension of Artin algebras, Queen Mary College Mathematical Notes. (London), 1971.

[CPS] E. Cline, B. Parshall and L. Scott. Finite dimensional algebras and highest weight categories, J. Reine Angew. Math. 391 (1988), 85-99. 
[DR , ] V. Dlab and C. M. Ringel, Quasi-hereditary algebras, Ill. J. Math. (to appear).

[DR $\left.{ }_{2}\right]$ _. Auslander algebras as quasi-hereditary algebras, J. London Math. Soc. (to appear).

[PS] B. Parshall and L. Scott, Derived categories, quasi-hereditary algebras and algebraic groups, Proc. Ottawa-Moosonee Workshop in Algebra, Carleton Univ. Notes No. 3 (1988).

[S] L. Scott, Simulating algebraic geometry with algebras I: the algebraic theory of derived categories, Amer. Math. Soc. Proc. Symp. Pure Math. 47 (Providence, 1988), 271-281.

Department of Mathematics, Carleton University, Ottawa, K1S 5B6, Canada

Fakultät für Mathematik, Universität Bielefeld, D-4800 Bielefeld 1, West Germany 